\title{
Global Attractor for Second-Order Nonlinear Evolution Differential Inclusions
}

\author{
Guangwang $\mathrm{Su}^{1,2,3}$ and Funing $\operatorname{Lin}$ (D) $^{2,3}$ \\ ${ }^{1}$ School of Science, Nanjing University of Science and Technology, Nanjing 210094, China \\ ${ }^{2}$ School of Information and Statistics, Guangxi University of Finance and Economics, Nanning 530003, China \\ ${ }^{3}$ Guangxi Key Laboratory of Cross-border E-commerce Intelligent Information Processing, \\ Guangxi University of Finance and Economics, Nanning 530003, China
}

Correspondence should be addressed to Funing Lin; toplin518@126.com

Received 20 January 2021; Revised 13 February 2021; Accepted 23 February 2021; Published 4 March 2021

Academic Editor: Ahmed Mostafa Khalil

Copyright (c) 2021 Guangwang Su and Funing Lin. This is an open access article distributed under the Creative Commons Attribution License, which permits unrestricted use, distribution, and reproduction in any medium, provided the original work is properly cited.

In this paper, we address the model of global attractor formulated in the form of evolution differential inclusions with second order in Banach spaces. Firstly, based on the fixed point theorem, the existence result of mild solutions is deduced. Then, by implementing the measure of noncompactness, the existence of global attractor associated with $m$-semiflow is validated. Finally, a concrete application of the main result is demonstrated to enhance the practical signification.

\section{Introduction}

Consider a Banach space $(E,\|\cdot\|)$. We attempt to handle the following problem:

$$
\left\{\begin{array}{l}
u^{\prime \prime}(t)-A u(t) \in F(t, u(t)), \quad t>0 \\
u(0)=u_{0}, \quad u \prime(0)=v_{0}
\end{array}\right.
$$

where $A$ is a closed, linear, and densely defined operator from $E$ to $E$ that generates a strongly continuous cosine family $\{C(t)\}_{t \geq 0} ; F:[0,+\infty) \times E \longrightarrow \mathscr{P}(E)$ is measurable and upper semicontinuous in terms of both first-order and second-order variables, and it also satisfies the natural growth condition.

The nonlinear evolution differential inclusions in Banach spaces have drawn more and more attention of a large number of experts and scholars during the past decades, see [1]. At present, there are substantial outcomes focusing on the existence of solutions for differential equations with second or higher order [2, 3]. For instance, semilinear evolution differential inclusions were explored by Cardinali and Rubbioni in [4]. Liu, Li, and Migórski et al. discussed the problems of one and second evolution differential variational inequalities, and they attained several existence results about the mild solution, see $[5,6]$ and references therein.

It is known that global attractor has played an important role in studying the asymptotic feature of solutions for diverse types of differential systems. Melnik, Valero, and Tran et al. were devoted to researching on global attractor of multivalued semiflows and differential inclusions, see [7-13] and references therein. In [10], the authors studied the global attractor of the following functional differential inclusions:

$$
\left\{\begin{array}{l}
u^{\prime}(t)-A u(t) \in F\left(u(t), u_{t}\right), \quad t>0, \\
u(s)=\varphi(s), \quad s \in[-h, 0],
\end{array}\right.
$$

where $u$ represents the state function and $u_{t}$ describes the history of the state function, that is, $u_{t}(s)=u(t+s)$ for $s \in[-h, 0]$. They proved the existences of solutions of system (2) and global attractor by applying noncompactness measure. Global attractor is a gradual state of solution set, and it is also an important research content of a dynamic system. Compared with the first-order differential inclusions, the cosine family generated by operator $A$ is more complex and more meaningful. There are relatively few 
studies on this type of second-order differential inclusions, so it is more important to study the attractor of the secondorder differential inclusions.

The first aim of the work is to explore the existence of solutions in terms of system (1) in infinite dimensional spaces. Secondly, we are devoted to show the existence of a global attractor for system (1). To our knowledge, there is currently a research gap in the aforementioned problem. However, it is not easy to establish a connection between mild solution and $m$-semiflows for second-order differential inclusions.

The work is structured as follows. Section 2 presents several relevant definitions involving noncompactness measures and $m$-semiflows with its global attractor. In Section 3, several sufficient conditions with respect to the mild solutions are invoked. In Section 4, the presence of a global attractor of system (1) is examined in Banach spaces. Finally, a concrete application is presented to emphasize the magnitude of the main results.

\section{Preliminaries}

This section recalls some fundamental definitions and properties of nonlinear analysis, for more details, see [14]. The symbol $\mathbb{R}$ (resp., $\mathbb{R}_{+}$) represents the set of real (resp., positive real) numbers. The strong and weak convergences of $\left\{u_{k}\right\}$ to $u$ are denoted by $u_{k} \longrightarrow u$ and $u_{k} \rightarrow u$, respectively.

Consider a Banach space $E$ whose dual space is denoted by $E^{*}$. Let $I=[0, T]$ with $0<T<\infty$ and let $L^{1}(I, E)$ be the Banach space of all (Bochner) integrable functions on $I$ endowed with the norm $\|f\|=\int_{I}\|f(s)\|_{E} \mathrm{~d} s$. The notations mentioned as follows will be employed:

$$
\begin{aligned}
\mathscr{P}_{f(c)}(E):=\{Q \subset E: Q \text { is nonempty, closed (convex) }\}, \\
\mathscr{P}_{\omega k(c)}(E):=\{Q \subset E: Q \text { is nonempty, weakly compact (convex) }\} .
\end{aligned}
$$

Definition 1. Suppose that $E_{1}$ and $E_{2}$ are Banach spaces. A multivalued mapping $F: E_{1} \longrightarrow \mathscr{P}\left(E_{2}\right)$ is referred to as follows:

(1) Upper semicontinuous (u.s.c), provided that, for an arbitrary closed set $Q$ in $E_{2}$,

$$
F^{-1}(Q)=\left\{e \in E_{1}: F(e) \cap Q \neq \varnothing\right\},
$$

is closed in $E_{1}$.

(2) Weakly upper semicontinuous (weakly u.s.c), if for arbitrary weakly closed set $Q$ in $E_{2}$,

$$
F^{-1}(Q)=\left\{e \in E_{1}: F(e) \cap Q \neq \varnothing\right\},
$$

is closed in $E_{1}$.

(3) Closed, if its graph $\Gamma_{F}=\left\{\left(e_{1}, e_{2}\right): e_{2} \in F\left(e_{1}\right)\right\}$ is closed in $E_{1} \times E_{2}$.

(4) Compact, if $F(D)$ is a relatively compact subset of $E_{2}$ for every bounded set $D$.
A weakly upper semicontinuous mapping with a weakly compact convex value has good properties. The following conclusion can be found in [15].

Lemma 1. Suppose that $E_{1}$ and $E_{2}$ are Banach spaces and $F: E_{1} \longrightarrow \mathscr{P}_{w k c}\left(E_{2}\right)$ is a mapping. Then, $F$ is weakly u.s.c if only and if for any sequence $\left\{u_{n}\right\}$ of $E_{1}$ satisfying $u_{n} \longrightarrow u_{0} \in E_{1}$ and $v_{n} \in F\left(u_{n}\right)$, it implies $v_{n} \rightarrow v_{0} \in F\left(u_{0}\right)$ up to a subsequence.

In the subsequent arguments, we consider the presence of mild solutions about system (1) from the viewpoint of evolution differential inclusions with second order. We only need to deal with the following differential equation:

$$
\left\{\begin{array}{l}
u^{\prime \prime}(t)=A u(t)+f(t), \quad t>0, \\
u(0)=u_{0}, \quad u^{\prime}(0)=v_{0},
\end{array}\right.
$$

where $f(t) \in F(t, u(t))$.

Definition 2. Assume that $\mathscr{L}(E)$ is the space of all bounded linear operators defined from $E$ to $E^{*}$. A mapping $C: \mathbb{R} \longrightarrow \mathscr{L}(E)$ is called a strongly continuous cosine family, provided that $C(0)=I_{0}\left(I_{0}\right.$ is an identity operator $)$ and $C\left(r_{1}+r_{2}\right)+C\left(r_{1}-r_{2}\right)=2 C\left(r_{1}\right) C\left(r_{2}\right)$ for every $r_{1}, r_{2} \in \mathbb{R}$.

Given a strongly continuous cosine family $C(t)$ with $t \in \mathbb{R}$. Then, its generator is an operator $A: E \longrightarrow E$ formulated as

$$
A u=\left.\frac{d^{2}}{d t^{2}} C(t) u\right|_{t=0}, \quad \forall u \in D(A),
$$

where the domain $D(A)=\left\{e \in E: C(t) e \in C^{2}(\mathbb{R}, E)\right\}$. By the definition of generator $A$, it is known that $A$ is closed, linear, and densely defined over $E$, see [2]. The sine operator $S: \mathbb{R} \longrightarrow \mathscr{L}(E)$, which is closely relevant to the cosine operator $C$, is expressed by

$$
S(t) u=\int_{0}^{t} C(s) u \mathrm{~d} s, \quad \forall t \in \mathbb{R}, u \in E
$$

Definition 3. A mapping $u \in C(I, E)$ is referred to as a mild solution of system (6), provided that there is an $f \in L^{1}(I, E)$ with $f(t) \in F(t, u(t))$ and

$$
u(t)=C(t) u_{0}+S(t) v_{0}+\int_{0}^{t} S(t-s) f(s) \mathrm{d} s, \quad \forall t \in I .
$$

Given an arbitrary bounded set $B$ in $E$, the Hausdorff measure of noncompactness (MNC, for short) is given by

$$
\chi(B)=\inf \{\varepsilon>0: B \text { has a finite } \varepsilon-\text { net }\} .
$$

Let $\Omega \subset L^{1}(I, E)$. We say that $\Omega$ is integrably bounded, if any element $f$ of $\Omega$ satisfies that $\|f(t)\| \leq g(t)$ for a.e. $t \in I$ with some $g \in L^{1}\left(I, \mathbb{R}_{+}\right)$. An integrably bounded $\Omega$ is said to be semicompact, provided that $\{\Omega(t)\}$ is relatively compact for a.e. $t \in I$.

The norm of product spaces $E \times E$ is represented by 


$$
\|(u, v)\|=\max \left\{\|u\|_{E},\|v\|_{E}\right\}, \quad \forall(u, v) \in E \times E .
$$

It is straightforward to see that, for any bounded set $B_{1} \times B_{2} \subset E \times E$,

$$
\tilde{\chi}\left(B_{1} \times B_{2}\right)=\max \left\{\chi\left(B_{1}\right), \chi\left(B_{2}\right)\right\},
$$

where $\tilde{\chi}$ is measure of noncompactness on $E \times E$.

Definition 4. Presume that $\chi$ is a Hausdorff measure of noncompactness and $0 \leq \kappa<1$. A multivalued mapping $F: X \longrightarrow \mathscr{P}_{k}(E)$ is referred to as $\kappa$-condensing, if

$$
\chi(F(B)) \leq \kappa \cdot \chi(B), \quad \forall B \subset E .
$$

The open (resp., closed) ball with centrum 0 and radius $r>0$ is denoted by $B_{r}(0)$ (resp., $\overline{B_{r}(0)}$ ). The fixed point theorem used in our main results is introduced in [16] as follows.

Lemma 2. Define two operators $\mathscr{A}: \overline{B_{r}(0)} \longrightarrow X$ and $\mathscr{B}: \overline{B_{r}(0)} \longrightarrow \mathscr{P}_{k c}(E)$ by coupling with the following properties:

(1) $\mathscr{A}$ is a contraction mapping with a single value and a coefficient $\lambda<1 / 2$

(2) $\mathscr{B}$ is compact and u.s.c

Then, each of the following conclusions holds:

(i) There exists an element $\omega \in \overline{B_{r}(0)} \backslash B_{r}(0)$ such that $\rho \omega \in \mathscr{A} \omega+\mathscr{B} \omega$ for some $\rho>1$

(ii) The operator inclusion $u \in \mathscr{A} u+\mathscr{B} u$ admits a solution in $\overline{B_{r}(0)}$

Next, we introduce related theories involving global attractors and m-semiflow, see $[8,10,11]$. Suppose that $\Gamma$ is a nontrivial subgroup of the additive group of $\mathbb{R}$ and $\Gamma_{+}=\Gamma \cap \mathbb{R}_{+}$.

Definition 5. A mapping $\Phi: \Gamma \times E \longrightarrow \mathscr{P}(E)$ is said to be a multivalued flow ( $m$-flow, in short) if the following conditions are guaranteed:

(1) $\Phi(0, u)=u$ for every $u \in E$

(2) $\Phi(t+s, u) \subset \Phi(t, \Phi(s, u))$ for any pair $t, s \in \Gamma$ and $u \in E$ with $\Phi(\tau, B)=\cup_{u \in B} \Phi(\tau, u)$

Remark 1. A mapping $\Phi: \Gamma_{+} \times E \longrightarrow \mathscr{P}(E)$ is referred to as an $m$-semiflow, if conditions (1) and (2) are satisfied for any $t_{1}, t_{1} \in \Gamma_{+}$.

A mapping $\Phi$ is said to be a strict $m$-semiflow if for every $t, s \in \Gamma_{+}, u \in E$, we have $\Phi(t+s, u)=\Phi(t, \Phi(s, u))$. A map $u(\cdot): \Gamma_{+} \longrightarrow E$ is called a trajectory of $m$-semiflow with respect to the initial condition $u(0)=u_{0}$, if $u(t+s) \in \Phi(t, u(s))$ for all $t, s \in \Gamma_{+}$.

Definition 6. Let $\Phi$ be an $m$-semiflow. A bounded set $B_{0} \subset E$ is referred to as an absorbing set for $\Phi$, provided that, for every bounded $B_{1} \subset E, \quad \gamma_{T\left(B_{1}\right)}^{(+)} \subset B_{0}$ holds for some $T=T\left(B_{1}\right) \geq 0$, where $\gamma_{T\left(B_{1}\right)}^{(+)}=\bigcup_{t \geq T\left(B_{1}\right)} \Phi\left(t, B_{1}\right)$.
For a given $e \in E$ and $B_{1}, B_{2} \subset E$, we define $d\left(e, B_{i}\right)=\inf _{x \in B_{i}}\{d(e, x)\}$. The metric excess of $B_{1}$ over $B_{2}$ is given as $\eta\left(B_{1}, B_{2}\right)=\sup _{e \in B_{1}}\left\{d\left(e, B_{2}\right)\right\}$. It is well known that the Hausdorff distance between $B_{1}$ and $B_{2}$ can be represented in the following way:

$$
d_{H}\left(B_{1}, B_{2}\right)=\max \left\{\eta\left(B_{1}, B_{2}\right), \eta\left(B_{2}, B_{1}\right)\right\} .
$$

Definition 7. Let $\Phi$ be an $m$-semiflow. A set $\Re$ is said to be a global attractor of $\Phi$, provided that the following conditions are satisfied:

(1) $\Re$ attracts any bounded set $B$ in $E$, namely, $\eta(\Phi(t, B), \mathfrak{R}) \longrightarrow 0$ as $t \longrightarrow+\infty$

(2) $\mathfrak{R}$ is negatively semi-invariant, namely, $\Re \subset \Phi(t, \mathfrak{R})$, for all $t \in \Gamma_{+}$

We now give an existence result about a global attractor for a given $m$-semiflow $\Phi$, see [11].

Lemma 3. Assume that a mapping $\Phi: \Gamma_{+} \times E \longrightarrow \mathscr{P}(E)$ satisfies the following conditions:

(i) For every $t \in \Gamma_{+}, u \longrightarrow \Phi(t, u)$ is u.s.c. with closed values

(ii) Ф has an absorbing set

(iii) $\Phi$ is asymptotically upper semicompact, i.e., for every bounded $B \subset E$ with bounded $\gamma_{T(B)}^{(+)}$for some positive value $T(B)$, any sequence $\left\{\xi_{n} \in E: \xi_{n} \in \Phi\left(t_{n}, B\right)\right\}$ is relatively compact as $t_{n} \longrightarrow+\infty$

Then, $\Phi$ admits a compact global attractor $\mathfrak{R}$ in $E$. In particular, $\mathfrak{R}$ is invariant if it is a strict $m$-semiflow, i.e., $\mathfrak{R}=\Phi(t, \mathfrak{R}), \forall t \in \Gamma_{+}$.

\section{The Existence Result of Mild Solutions}

This section is based on the idea of [3] for nonlinear evolution hemivariational inequalities with second order, which is adapted for evolution differential inclusions in our context. We will handle problem (6) under the following basic assumptions:

(A) The operator $A: D(A) \subset E \longrightarrow E$ is a generator of a cosine operator $\{C(t)\}_{t \in \mathbb{R}}$, and $\|C(t)\|,\|S(t)\| \leq M_{A} e^{w|t|}$ for some $M_{A} \geq 1$ and $w \geq 0$. Moreover, $S(t)$ is compact for arbitrary $t \in I$.

(F) The multivalued mapping $F: I \times E \longrightarrow \mathscr{P}_{k c}(E)$ is u.s.c., and there are $a \in L^{2}\left(I, \mathbb{R}_{+}\right)$and $b>0$ with

$$
\begin{aligned}
\|F(t, u)\| & =\sup \{\|\xi\|: \xi \in F(t, u)\} \\
& \leq a(t)+b\|u\| \quad \text { for every }(t, u) \in I \times E .
\end{aligned}
$$

By combining the uniform boundedness principle with the assumption $(A)$, it is inferred that $C(t)$ (resp., $S(t)$ ) is uniformly bounded on $I$ subject to some upper-bound $M_{C}$ (resp., $M_{S}$ ). We set $M=\max \left\{M_{C}, M_{S}\right\}$.

In the sequel, let a multivalued operator $\mathscr{P}_{F}: C(I, E) \longrightarrow \mathscr{P}\left(L^{2}(I, E)\right)$ be 


$$
\mathscr{P}_{F}(u)=\left\{f \in L^{2}(I, E): f(t) \in F(t, u(t)) \text { for a.e. } t \in I\right\} .
$$

It is well known that $F(t, u)$ has a measurable selection for every $u \in C(I, E)$ and a.e. $t \in I$ under the assumption $F$, which means that $\mathscr{P}_{F}$ is nonempty and well-defined.

We also construct a multivalued operator $\mathscr{B}: C(I, E) \longrightarrow C(I, E)$ by

$$
\mathscr{B}(u)=\left\{\phi \in C(I, E): \phi(t)=\int_{0}^{t} S(t-s) f(s) \mathrm{d} s, \quad f \in \mathscr{P}_{F}(u)\right\} .
$$

Set $\mathscr{W}: L^{2}(I, E) \longrightarrow C(I, E)$ by

$$
\mathscr{W}(f)(t)=\int_{0}^{t} S(t-s) f(s) \mathrm{d} s .
$$

Lemma 4. Assume that $(A)$ and $(F)$ hold. Then, for an arbitrary sequence $\left\{f_{n}\right\}_{n=1}^{\infty} \subset L^{2}(I, E)$ with semicompactness, the sequence $\left\{\mathscr{W} f_{n}\right\}_{n=1}^{\infty}$ is relatively compact. In particular, if $f_{n} \rightarrow f_{0}$, then $\mathscr{W} f_{n} \longrightarrow \mathscr{W} f_{0}$.

Proof. It is shown by analogizing the proof of Theorem 2 of [4].

Aiming to validate the presence of solutions for system (1), we now clarify a property of the multivalued operator $\mathscr{B}$, which is inspired by Lemma 5 of [3].

Lemma 5. Assume that $(A)$ and $(F)$ hold. Then, the multivalued mapping $\mathscr{B}: C(I, E) \longrightarrow C(I, E)$ is compact and u.s.c. with convex and compact values.

Proof. Note that the operator $\mathscr{B}$ has convex values for any $u \in C(I, E)$ due to the convexity of $\mathscr{P}_{F}$. The verification process will be divided into four steps as follows:

Step 1: $\mathscr{B}$ is a bounded operator on $C(I, E)$. Given $u \in B_{r}(0)$ and $\phi \in \mathscr{B}(u)$, from $(F)$ and Hölder inequality, it follows that

$$
\begin{aligned}
\|\phi(t)\|_{E} & \leq \int_{0}^{t}\|S(t-s) f(s)\|_{E} \mathrm{~d} s \leq M \int_{0}^{t} a(s)+b\|u(s)\|_{E} \mathrm{~d} s \\
& \leq M\left(\|a\|_{L^{2}\left(I, \mathbb{R}_{+}\right)} \sqrt{T}+b r T\right) .
\end{aligned}
$$

Hence, $\mathscr{B}\left(B_{r}(0)\right)$ is a bounded subset of $C(I, E)$, i.e., the operator $\mathscr{B}$ is a bounded operator.

Step 2: $\left\{\mathscr{B}(u): u \in B_{r}(0)\right\}$ is equicontinuous. Presume that $0<t_{1}<t_{2} \leq T, \delta>0$ is sufficiently small, then

$$
\begin{aligned}
& \left\|\phi\left(t_{2}\right)-\phi\left(t_{1}\right)\right\|_{E}=\left\|\int_{0}^{t_{2}} S\left(t_{2}-s\right) f(s) \mathrm{d} s-\int_{0}^{t_{1}} S\left(t_{1}-s\right) f(s) \mathrm{d} s\right\|_{E} \\
& \leq \int_{0}^{t_{1}}\left\|\left[S\left(t_{2}-s\right)-S\left(t_{1}-s\right)\right] f(s)\right\| \mathrm{d} s+\int_{t_{1}}^{t_{2}}\left\|S\left(t_{2}-s\right) f(s)\right\| d s \\
& \leq \int_{0}^{t_{1}}\left\|\left[S\left(t_{2}-s\right)-S\left(t_{1}-s\right)\right]\right\|[a(s)+b r] \mathrm{d} s+M \int_{t_{1}}^{t_{2}}[a(s)+b r] \mathrm{d} s \\
& \leq \sup _{s \in\left[0, t_{1}-\delta\right]}\left\|\left[S\left(t_{2}-s\right)-S\left(t_{1}-s\right)\right]\right\|\left(\|a\|_{L^{2}\left(I, \mathbb{R}_{+}\right)} \sqrt{T}+b r T\right) \\
& \quad+M\left[\|a\|_{L^{2}\left(I, \mathbb{R}_{+}\right)}\left(2 \sqrt{\delta}+\sqrt{t_{2}-t_{1}}\right)+b r\left(2 \delta+t_{2}-t_{1}\right)\right] .
\end{aligned}
$$

From the continuity of the sine operator $S(t)$, we get easily that the above inequality is independent of $u$ and it converges to 0 as $t_{2} \longrightarrow t_{1}$. This implies the equicontinuity of $\left\{\mathscr{B}(u): u \in B_{r}(0)\right\}$ in $C(I, E)$.

Step 3: $\Pi(t)=\left\{\phi(t): \phi \in \mathscr{B}\left(B_{r}(0)\right)\right\}$ is relatively compact in $E$ for a.e. $t \in I$. Firstly, the compactness of $\Pi(0)=\{0\}$ is trivially checked. Then, fix $t \in(0, T]$. For any $u \in B_{r}(0)$ and $\phi \in \mathscr{B}\left(B_{r}(0)\right)$, there exists $f \in \mathscr{P}_{F}(u)$ with

$$
\phi(t)=\int_{0}^{t} S(t-s) f(s) \mathrm{d} s .
$$

For every $\varepsilon \in(0, t)$, we define

$$
\phi^{\varepsilon}(t)=\int_{0}^{t-\varepsilon} S(t-s) f(s) \mathrm{d} s=S(\varepsilon) \int_{0}^{t-\varepsilon} S(t-s-\varepsilon) f(s) \mathrm{d} s .
$$

It can be seen that the compactness of $S(\cdot)$ with the boundedness of $\int_{0}^{t-\varepsilon} S(t-s-\varepsilon) f(s) \mathrm{d} s$ together assures that $\Pi_{\varepsilon}(t)=\left\{\phi^{\varepsilon}(t): \phi \in \mathscr{B}\left(B_{r}(0)\right)\right\}$ is relatively compact in $E$. Furthermore, we have

$$
\begin{aligned}
& \left\|\phi(t)-\phi^{\varepsilon}(t)\right\|_{E} \leq M \int_{t-\varepsilon}^{t} a(s)+b\|u(s)\|_{E} \mathrm{~d} s \\
& \leq M\left(\|a\|_{L^{2}\left(I, \mathbb{R}_{+}\right)} \sqrt{\varepsilon}+b r \varepsilon\right) .
\end{aligned}
$$

Thereby, $\Pi(t)(t>0)$ is totally bounded, and thus it is relatively compact in $E$.

To this end, by applying Ascoli-Arzelà theorem and taking account of Steps 1-2, it can be verified that the mapping $\mathscr{B}$ is compact.

Step 4: $\mathscr{B}$ has a closed graph. In fact, let $\left\{u_{n}\right\},\left\{\phi_{n}\right\} \subset C(I, E), u_{n} \longrightarrow \bar{u}, \phi_{n} \in \mathscr{W}^{\circ} \circ \mathscr{P}_{\mathrm{F}}\left(\mathrm{u}_{\mathrm{n}}\right), \quad$ and $\phi_{n} \longrightarrow \bar{\phi}$. Choose any sequence $f_{n} \subset L^{2}(I, E)$ such that $f_{n} \in \mathscr{P}_{F}\left(u_{n}\right), \phi_{n}=\mathscr{W}\left(f_{n}\right)$. From $(F)$, we conclude that the sequence $f_{n}$ is integrable bounded. Hence, $f_{n}$ is weakly compact in $L^{2}(I, E)$. It is rational to presume that $f_{n} \rightarrow \bar{f}$. From Lemma 4 , we have 


$$
\phi_{n}=\mathscr{W}\left(f_{n}\right) \longrightarrow \mathscr{W}(\bar{f})=\bar{\phi} .
$$

Besides, $\bar{f} \in \mathscr{P}_{F}(\bar{u})$ due to Lemma 1 . Therefore, $\mathscr{B}$ has a closed graph.
Theorem 1. Presume that $(A)$ and $(F)$ hold. Then, there is at least one mild solution related to system (6) for each pair of initial values $u_{0}, v_{0} \in E$.

Proof. Construct a multivalued mapping $\mathscr{F}: C(I, E) \longrightarrow C(I, E)$ by

$$
\mathscr{F}(u)=\left\{\varphi \in C(I, E): \varphi(t)=C(t) u_{0}+S(t) v_{0}+\int_{0}^{t} S(t-s) f(s) \mathrm{d} s, \quad f \in \mathscr{P}_{F}(u)\right\} .
$$

$$
\|u(t)\|_{E} \leq d e^{M b t}
$$

For convenience, we write $\mathscr{F}=\mathscr{A}+\mathscr{B}$, where $\mathscr{B}$ is given by (17) and $\mathscr{A}$ is defined as

$$
\mathscr{A}(u)=C(t) u_{0}+S(t) v_{0}, \quad t \in I .
$$

Obviously, $u$ is a fixed point of $\mathscr{F}$ if only and if it is a mild solution of system (6). According to Lemma 2, we need to prove that (i) of Lemma 2 is not satisfied.

In fact, suppose that $\rho u \in \mathscr{A} u+\mathscr{B} u$ with $\rho>1$ and there exists $\eta \in \mathscr{P}_{F}(u)$ with

$$
\rho u(t)=C(t) u_{0}+S(t) v_{0}+\int_{0}^{t} S(t-s) \eta(s) \mathrm{d} s .
$$

This yields that

$$
\begin{aligned}
\|u(t)\|_{E} & \leq\left\|C(t) u_{0}\right\|_{E}+\left\|S(t) v_{0}\right\|_{E}+\left\|\int_{0}^{t} S(t-s) \eta(s) \mathrm{d} s\right\|_{E} \\
& \leq M\left\|u_{0}\right\|_{E}+M\left\|v_{0}\right\|_{E}+M \int_{0}^{t} a(s)+b\|u(s)\|_{E} \mathrm{~d} s \\
& \leq d+M b \int_{0}^{t}\|u(s)\|_{E} \mathrm{~d} s,
\end{aligned}
$$

This implies

$$
\|u\|_{C(I, E)} \leq d e^{M b T}=: r .
$$

Furthermore, we write

$$
Q_{r}=\left\{u \in C(I, E):\|u\|_{C(I, E)}<r+1\right\} .
$$

Obviously, $Q_{r}$ is open in $C(I, E)$. Utilizing Lemma 5 , we know that $\mathscr{B}:{\overline{Q_{r}}}^{\longrightarrow} \mathscr{P}_{k c}(E)$ is compact and u.s.c. We also see easily that $\mathscr{A}: \overline{Q_{r}} \longrightarrow E$ is a contraction mapping with single value and coefficient $\lambda<1 / 2$. Therefore, according to the choice of $\overline{Q_{r}}$, none of $u \in E$ with $\|u\|=r$ ensures that $\lambda u \in \mathscr{A} u+\mathscr{B} u$ with some $\rho>1$.

Applying Lemma 3, one knows that $\mathscr{F}$ has a fixed point due to all the conditions being fulfilled. Hence, there is a mild solution for system (6).

Next, let us discuss some features of the solution set. For every $T>0$, we say that $\pi_{T}$ is a truncation operator form $C([0,+\infty), E)$ to $C([0, T], E)$ if for any $z \in C([0,+\infty), E)$, we have $\pi_{T}(z) \in C([0, T], E)$. Put

where $d=M\left\|u_{0}\right\|_{E}+M\left\|v_{0}\right\|_{E}+M\|a\|_{L^{2}\left(I, \mathbb{R}_{+}\right)} \sqrt{T}$. Employing Gronwall inequality, one gets

$$
\Sigma(\xi)=\left\{u \in C([0,+\infty), E): u \text { is a mild solution of }(1.1) \text { on }[[0, T]], \xi=\left(u_{0}, v_{0}\right)\right\}
$$

where $u(0)=u_{0}, u^{\prime}(0)=v_{0}$. Obviously, $\pi_{T}^{\circ} \sum(\xi)=\operatorname{Fix}(\mathscr{F})$ and $\pi_{T}{ }^{\circ} \Sigma(\xi) \subset S(\cdot) \xi+\mathscr{W}^{\circ} \mathscr{P}_{\mathrm{F}}\left(\pi_{\mathrm{T}}{ }^{\circ} \Sigma(\xi)\right)$. Here, $\operatorname{Fix}(\mathscr{F})$ stands for the solution set of the fixed points of system (6).

Lemma 6. Let $U=E \times E$ and all the assumptions of Theorem 1 be fulfilled. Then, $\pi_{T}^{\circ} \Sigma\left(\xi_{n}\right)$ is relatively compact in $C([0, T], E)$ for arbitrary sequence $\xi_{n} \longrightarrow \xi$ in $U$. Especially, for every $\xi \in U, \pi_{T}^{\circ} \Sigma(\xi)$ is compact in $C([0, T], E)$.

Proof. It is verified by analogizing the proof of Lemma 3.7 of [8]. follows:

$$
\begin{aligned}
& \Phi: \mathbb{R}_{+} \times U \longrightarrow \mathscr{P}(U) \\
& \Phi(t, \xi)=\left\{(u(t), u(t)): x \text { is a mild solution of }(1.1), \xi=\left(u_{0}, v_{0}\right)\right\} .
\end{aligned}
$$

By applying a similar discussion in [11], we can obtain that $\Phi$ is a strict $m$-semiflow, i.e., for arbitrary $t, s \in \mathbb{R}_{+}$and $\xi \in U$, it holds that

$$
\Phi(t+s, \xi)=\Phi(t, \Phi(s, \xi)) .
$$

Lemma 7. Assume that all the hypotheses of Theorem 1 are fulfilled. For all $t>0, \Phi(t, \cdot)$ is u.s.c. with compact values.

Proof. It is verified by analogizing the proof of Lemma 3.8 of [8]. 


\section{The Existence Result of Global Attractors}

In the sequel, we will present a severe validation for the existence of a global attractor of $m$-semiflow $\Phi$ induced by system $(1)$. The conditions $(A)$ and $(F)$ are replaced by $\left(A^{\prime}\right)$ and $\left(F^{\prime}\right)$ as follows, respectively:

$\left(A^{\prime}\right)$ The cosine and sine functions are exponentially stable with exponent $\alpha>0$, that is, there exist $M_{0} \geq 1$ and $\alpha \geq 0$ with

$$
\|C(t)\|_{\mathscr{L}(E)} \leq M_{0} e^{-\alpha t},\|S(t)\|_{\mathscr{L}(E)} \leq M_{0} e^{-\alpha t}, \quad \forall t>0 .
$$

$\left(F^{\prime}\right)$ The multivalued mapping $F: \mathbb{R}_{+} \times E \longrightarrow \mathscr{P}_{k c}(E)$ is u.s.c. and satisfies the following conditions:

(1) There exists $a_{1} \in L^{2}\left(\mathbb{R}_{+}, \mathbb{R}_{+}\right)$and $b_{1}>0$, ensuring that

$$
\begin{aligned}
& \|F(t, x)\|=\sup \{\|\xi\|: \xi \in F(t, u)\} \\
& \quad \leq a_{1}(t)+b_{1}\|u\|, \quad \forall(t, u) \in \mathbb{R} \times E .
\end{aligned}
$$

(2) There exists $q \in L^{2}\left(\mathbb{R}_{+}, \mathbb{R}_{+}\right)$ensuring that, for every bounded $\Omega$ in $E$,

$\chi(F(t, \Omega)) \leq q(t) \chi(\Omega) \quad$ for almost all $t \in R_{+}$.

Given $T>0$, for convenience, the translation operator $\Phi(T, \cdot)$ is defined by $\Phi_{T}$. The following condensing property of $\Phi_{T}$ is an essential result to reveal that $\Phi$ is asymptotically upper semicompact.

Lemma 8. Suppose that $\left(A^{\prime}\right)$ and $\left(F^{\prime}\right)$ are satisfied. Then, there are $\zeta \in[0,1)$ and $T_{0}>0$, guaranteeing that, for all $T \geq T_{0}$ and all bounded $B \subset U$, it is inferred that

$$
\tilde{\chi}\left(\Phi_{T}(B)\right) \leq \zeta \cdot \widetilde{\chi}(B),
$$

whenever

$$
\alpha-4 M_{0}\|q\|_{L^{2}\left(\mathbb{R}_{+}, \mathbb{R}_{+}\right)}>0 .
$$

Proof. Let $U=E \times E, B=B_{1} \times B_{2}$ be bounded in $U$. Denote $D=\Sigma(B)$, we obtain

$$
D(t) \subset C(t) B_{1}+S(t) B_{2}+\int_{0}^{t} S(t-s) \mathscr{P}_{F}(D)(s) \mathrm{d} s, t>0 .
$$

Therefore, we get

$$
\begin{aligned}
\tilde{\chi}\left(\Phi_{t}(B)\right)= & \chi(D(t)) \leq M_{0} e^{-\alpha t} \chi\left(B_{1}\right)+M_{0} e^{-\alpha t} \chi\left(B_{2}\right) \\
& +\chi\left(\int_{0}^{t} S(t-s) \mathscr{P}_{F}(D)(s) \mathrm{d} s\right) \\
\leq & 2 M_{0} e^{-\alpha t} \widetilde{\chi}(B)+4 M_{0} \int_{0}^{t} e^{-\alpha(t-s)} \chi\left(\mathscr{P}_{F}(D)(s)\right) \mathrm{d} s \\
\leq & 2 M_{0} e^{-\alpha t} \widetilde{\chi}(B)+4 M_{0} e^{-\alpha t} \int_{0}^{t} e^{\alpha s} q(s) \chi(D(s)) \mathrm{d} s \\
\leq & 2 M_{0} e^{-\alpha t} \widetilde{\chi}(B)+4 M_{0}\|q\|_{L^{2}}\left(\mathbb{R}_{+}, \mathbb{R}_{+}\right)^{-\alpha t} \\
& \int_{0}^{t} e^{\alpha s} \chi(D(s)) \mathrm{d} s .
\end{aligned}
$$

Hence,

$e^{\alpha t} \chi(D(t)) \leq 2 M_{0} \tilde{\chi}(B)+4 M_{0}\|q\|_{L^{2}\left(\mathbb{R}_{+}, \mathbb{R}_{+}\right)} \int_{0}^{t} e^{\alpha s} \chi(D(s)) \mathrm{d} s$.

Applying the Gronwall inequality, we have

$$
e^{\alpha t} \chi(D(t)) \leq 2 M_{0} e^{4 M_{0}\|q\|_{L^{2}\left(\mathbb{R}_{+}, \mathbb{R}_{+}\right)}} \tilde{\chi}(B) .
$$

Equivalently,

$$
\chi(D(t)) \leq 2 M_{0} e^{-\left(\alpha-4 M_{0}\|q\|_{L^{2}\left(\mathbb{R}_{+}, \mathbb{R}_{+}\right)}\right) t} \widetilde{\chi}(B) .
$$

Note that $\tilde{\chi}\left(\Phi_{t}(B)\right)=\chi(D(t))$, we get

$$
\tilde{\chi}\left(\Phi_{T}(B)\right) \leq \zeta_{t} \cdot \tilde{\chi}(B),
$$

where $\zeta_{t}=2 M_{0} e^{-\left(\alpha-4 M_{0}\|q\|_{L^{2}\left(\mathbb{R}_{+}, \mathbb{R}_{+}\right) t}\right.}$.

Taking $T_{0}>\ln 2 M_{0} / \alpha-4 M_{0}\|q\|_{L^{2}\left(\mathbb{R}_{+}, \mathbb{R}_{+}\right)}$and $\zeta=\zeta_{T_{0}}$, we conclude the proof.

Lemma 9. Presume that $\left(A^{\prime}\right)$ and $\left(F^{\prime}\right)$ are fulfilled. Then, $\Phi$ is asymptotically upper semicompact.

Proof. Let $U=E \times E$ and $B=B_{1} \times B_{2}$ be a bounded subset of $U$ and $\Delta(B)$ be a collection consisted of sequences $\xi_{n}$ with $\xi_{n} \in \Phi\left(t_{n}, B\right), t_{n} \longrightarrow \infty$. Put

$$
\eta=\sup \{\tilde{\chi}(Q): Q \in \Delta(B)\} .
$$

We claim that $\eta=0$ by using reduction. Assume that there exists $Q_{\theta}=\left\{\xi_{n} \in \Delta(B)\right\}$ such that

$$
\chi\left(Q_{\theta}\right)>\eta-\theta, \quad \forall \theta \in(0,(1-\zeta) \eta),
$$

where $\zeta$ is the same as in Lemma 8. Choose $T>0$ as described in Lemma 8; then, there exists $m_{n} \in \mathbb{N}$ such that $t_{n}=m_{n} T+$ $r_{n}, r_{n} \in[0, T)$ for any $t_{n} \in(T, \infty)$. Let $\tau_{n}=\left(m_{n}-1\right) T+r_{n}$. Then, 


$$
\xi_{n} \in \Phi\left(t_{n}, B\right)=\Phi\left(T+\tau_{n}, B\right)=\Phi_{T}\left(\Phi\left(\tau_{n}, B\right)\right) .
$$
obtain

By taking $\sigma_{n} \in \Phi\left(\tau_{n}, B\right)$ such that $\xi_{n} \in \Phi\left(T, \sigma_{n}\right)$, we

$$
\tilde{\chi}\left(Q_{\theta}\right)=\tilde{\chi}\left(\xi_{n}\right) \leq \tilde{\chi}\left(\Phi_{t}\left(\sigma_{n}\right)\right) \leq \zeta \widetilde{\chi}\left(\sigma_{n}\right) \leq \zeta \eta<\eta-\theta .
$$

This is a contradiction.

Lemma 10. Presume that $\left(A^{\prime}\right)$ and $\left(F^{\prime}\right)$ are fulfilled. If $\alpha>b M_{0}$, then an absorbing set exists for $m$-semiflow $\Phi$.
Proof. Let $t>0$ and $B=B_{1} \times B_{2}$ be bounded in $U=E \times E$. Then, for each $\xi=\left(u_{0}, v_{0}\right) \in U$ and $u \in \Sigma(\xi)$, there is $M_{B}>0$ with

$$
\left\{\begin{array}{l}
u(t)=C(t) u_{0}+S(t) v_{0}+\int_{0}^{t} S(t-s) f(s) \mathrm{d} s, \\
\left\|u_{0}\right\| \leq M_{B},\left\|v_{0}\right\| \leq M_{B},
\end{array}\right.
$$

for some $f \in \mathscr{P}_{F}(u)$. The combination of Hölder inequality, $\left(A^{\prime}\right)$ and the part (1) of $\left(F^{\prime}\right)$ gives

$$
\begin{aligned}
& \|u(t)\|_{X} \leq 2 M_{B} M_{0} e^{-\alpha t}+M_{0} \int_{0}^{t} e^{-\alpha(t-s)}\left[a_{1}(s)+b_{1}\|u(s)\|_{E}\right] \mathrm{d} s \\
& \leq 2 M_{B} M_{0} e^{-\alpha t}+M_{0} \int_{0}^{t} e^{-\alpha(t-s)} a_{1}(s) \mathrm{d} s+b_{1} M_{0} \int_{0}^{t} e^{-\alpha(t-s)}\|u(s)\|_{E} \mathrm{~d} s . \\
& \leq 2 M_{B} M_{0} e^{-\alpha t}+M_{0} e^{-\alpha t}\left[\left\|a_{1}\right\|_{L^{2}}\left(\mathbb{R}_{+}, \mathbb{R}_{+}\right) \frac{1}{\sqrt{2 \alpha}} e^{\alpha t}\right]+b_{1} M_{0} \int_{0}^{t} e^{-\alpha(t-s)}\|u(s)\|_{E} \mathrm{~d} s \\
& \leq 2 M_{B} M_{0} e^{-\alpha t}+M_{0}\left[\left\|a_{1}\right\|_{L^{2}\left(\mathbb{R}_{+}, \mathbb{R}_{+}\right)} \frac{1}{\sqrt{2 \alpha}}\right]+b_{1} M_{0} \int_{0}^{t} e^{-\alpha(t-s)}\|u(s)\|_{E} \mathrm{~d} s .
\end{aligned}
$$
obtain

Putting $c_{1}=M_{0}\left[\left\|a_{1}\right\|_{L^{2}\left(\mathbb{R}_{+}, \mathbb{R}_{+}\right)} 1 / \sqrt{2 \alpha}\right]$ and $c_{2}=b_{1} M_{0}$, we

The employment of Gronwall inequality yields

$$
e^{\alpha t}\|u(t)\|_{E} \leq 2 M_{B} M_{0}+c_{1} e^{\alpha t}+c_{2} \int_{0}^{t} e^{\alpha s}\|u(s)\|_{X} \mathrm{~d} s .
$$

$$
\begin{aligned}
e^{\alpha t}\|u(t)\|_{E} & \leq 2 M_{B} M_{0}+c_{1} e^{\alpha t}+c_{2} \int_{0}^{t}\left[2 M_{B} M_{0}+c_{1} e^{\alpha s}\right] e^{c_{2}(t-s)} \mathrm{d} s \\
& \leq 2 M_{B} M_{0}+c_{1} e^{\alpha t}+c_{2}\left[\int_{0}^{t} 2 M_{B} M_{0} e^{c_{2}(t-s)} \mathrm{d} s+\int_{0}^{t} c_{1} e^{\alpha s+c_{2} t-c_{2} s} \mathrm{~d} s\right] \\
& \leq 2 M_{B} M_{0}+c_{1} e^{\alpha t}+2 M_{B} M_{0}\left(e^{c_{2}}-1\right)+\frac{c_{1} c_{2}}{\alpha-c_{2}}\left[e^{\alpha t}-e^{c_{2} t}\right] .
\end{aligned}
$$

This implies

$$
\begin{aligned}
\|u(t)\|_{E} \leq & 2 M_{B} M_{0} e^{-\alpha t}+c_{1}+2 M_{B} M_{0} e^{\left(c_{2}-\alpha\right) t} \\
& +\frac{c_{1} c_{2}}{\alpha-c_{2}}\left[1-e^{\left(c_{2}-\alpha\right) t}\right] .
\end{aligned}
$$

The above inequality holds subject to the ball with the radius $R$ as

$$
R=c_{1}+\frac{c_{1} c_{2}}{\alpha-c_{2}}+1
$$
$\Phi$.

Moreover, it is actually an absorbing set in terms of

Theorem 2. Assume that $\left(A^{\prime}\right)$ and $\left(F^{\prime}\right)$ are fulfilled. Then, the $m$-semiflow $\Phi$ induced by system (1) has a compact global attractor, if the following holds:

$$
\min \left\{\alpha-4 M_{0}\|q\|_{L^{2}\left(\mathbb{R}_{+}, \mathbb{R}_{+}\right)}, \alpha-b_{1} M_{0}\right\}>0
$$

Proof. By Lemmas 7, 9, and 10, we obtain that all the conditions of Lemma 3 are satisfied. Therefore, the $m$-semiflow $\Phi$ admits a compact global attractor. 


\section{An Illustrative Example}

In this section, a concrete example is illustrated for the application of evolution partial different equations.
Take an open set $\Omega$ which is bounded in $\mathbb{R}^{n}$ and denote its boundary by $\partial \Omega$. The following system is worthy of survey:

$$
\left\{\begin{array}{l}
Z_{t t}(t, u)-\Delta Z(t, u)=f(t, u), \quad t>0, u \in \Omega, \\
f(t, u)=\lambda f_{1}(t, Z(t, u))+(1-\lambda) f_{2}(t, Z(t, u)), \quad \lambda \in[0,1], t>0, u \in \Omega, \\
Z(t, u)=0, \quad t \geq 0, u \in \partial \Omega, \\
Z(0, u)=Z_{0}(u), \quad Z_{t}(0, u)=Z_{1}(u),
\end{array}\right.
$$

where $f_{1}, f_{2}: \mathbb{R}_{+} \times \mathbb{R} \longrightarrow \mathbb{R}$ are continuous functions. This system describes a classical wave equation with a free bounded issue [1].

Put $E=L^{2}(\Omega)$. The multivalued mapping $F: \mathbb{R}_{+} \times E \longrightarrow \mathscr{P}(E)$ is described as

$$
F(\widetilde{Z})(t)=\left\{\lambda f_{1}(t, \widetilde{Z}(t))+(1-\lambda) f_{2}(t, \widetilde{Z}(t)): \lambda \in[0,1]\right\} .
$$

Then, (57) can be reformulated as

$$
\left\{\begin{array}{l}
Z^{\prime \prime}(t)-A Z(t) \in F(t, Z(t)), \quad t \geq 0, \\
Z(0)=Z_{0}, \quad Z_{t}(0)=Z_{1},
\end{array}\right.
$$

in which $A=\Delta, D(A)=H^{2}(\Omega) \cap H_{0}^{1}(\Omega)$, and $Z(t) \in E$.

Suppose that there exists $a_{1}, a_{2} \in L^{2}(\Omega)$ and positive constants $b_{1}$ and $b_{2}$ such that

$$
\begin{array}{r}
\left|f_{1}(t, p)\right| \leq a_{1}(t)+b_{1}|p|, \\
\left|f_{2}(t, p)\right| \leq a_{2}(t)+b_{2}|p|, \quad \forall x \in \Omega, p \in \mathbb{R} .
\end{array}
$$

Note that the mapping $F$ has convex, closed, and compact values. In addition,

$$
\|F(t, \widetilde{Z})\| \leq \max \left\{a_{1}(t), a_{2}(t)\right\}+\max \left\{b_{1}, b_{2}\right\}\|\widetilde{Z}\|_{X} .
$$

By the continuity of $f_{1}$ and $f_{2}$, we can obtain that there is a closed graph for F. Furthermore, by exploiting Lebesgue dominated convergence theorem, one can verify the quasicompactness of $F$. Therefore, $F$ is u.s.c., and $\left(F^{\prime}\right)(1)$ is testified. If $S(\cdot)$ is exponentially stable and compact, then $\left(A^{\prime}\right)$ and $\left(F^{\prime}\right)(2)$ is also satisfied. This indicates the presence of a global attractor, which belongs to the $m$-semiflow induced by (57).

\section{Data Availability}

The data used to support the findings of this study are available within the article.

\section{Conflicts of Interest}

The authors declare that there are no conflicts of interest.

\section{Acknowledgments}

This work was supported by the National Natural Science Foundation of China (Grant nos. 11761011 and 71862003),
Guangxi Natural Science Foundation (Grant no. 2020GXNSFAA297010), Guangxi College Young and Middle-aged Teachers Basic Ability Promotion Project (Grant no. 2019KY0669) and Collaborative Innovation Center for Integration of Terrestrial and Marine Economies.

\section{References}

[1] V. Barbu, Analysis and Control of Nonlinear Infinite Dimensional Systems, Academic Press, Boston, MA, USA, 1993.

[2] H. O. Fattorini, Second Order Linear Differential Equations in Banach Spaces, North-Holland Publishing, Amsterdam, The Netherlands, 1985.

[3] X. Li, Z. Liu, and S. Migórski, “Approximate controllability for second order nonlinear evolution hemivariational inequalities," Electronic Journal of Qualitative Theory of Differential Equations, vol. 100, no. 100, pp. 1-16, 2015.

[4] T. Cardinali and P. Rubbioni, "On the existence of mild solutions of semilinear evolution differential inclusions," Journal of Mathematical Analysis and Applications, vol. 308, no. 2, pp. 620-635, 2005.

[5] Z. Liu, X. Li, and D. Motreanu, "Approximate controllability for nonlinear evolution hemivariational inequalities in Hilbert spaces," SIAM Journal on Control and Optimization, vol. 53, no. 5, pp. 3228-3244, 2015.

[6] Z. Liu, S. Zeng, and D. Motreanu, "Evolutionary problems driven by variational inequalities," Journal of Differential Equations, vol. 260, no. 9, pp. 6787-6799, 2016.

[7] C. T. Anh, N. M. Chuong, and T. D. Ke, "Global attractor for the m-semiflow generated by a quasilinear degenerate parabolic equation," Journal of Mathematical Analysis and Applications, vol. 363, no. 2, pp. 444-453, 2010.

[8] N. T. V. Anh and T. D. Ke, "On the differential variational inequalities of parabolic-elliptic type," Mathematical Methods in the Applied Sciences, vol. 40, pp. 4683-4695, 2017.

[9] S. Ha, H. Liu, and S. Li, "Adaptive fuzzy backstepping control of fractional-order chaotic systems with input saturation," Journal of Intelligent \& Fuzzy Systems, vol. 37, no. 5, pp. 6513-6525, 2019.

[10] T. D. Ke and D. Lan, "Global attractor for a class of functional differential inclusions with Hille-Yosida operators," Nonlinear Analysis: Theory, Methods \& Applications, vol. 103, pp. 72-86, 2014.

[11] S. M. Valery and J. Valero, "On attractors of multivalued semiflows and differential inclusions," Set-Valued Analysis, vol. 6, pp. 83-111, 1998.

[12] Y. Zhou, H. Liu, J. Cao, and S. Li, "composite learning fuzzy synchronization for incommensurate fractional-order chaotic systems with time-varying delays," International journal of 
adaptive control and signal processing, vol. 33, no. 12, pp. 1739-1758, 2019.

[13] Y. Zhou, H. Wang, and H. Liu, "Generalized function projective synchronization of incommensurate fractional-order chaotic systems with inputs saturation," International Journal of Fuzzy Systems, vol. 21, no. 3, pp. 823-836, 2019.

[14] M. Kamenskii, V. Obukhovskii, and P. Zecca, Condensing Multivalued Maps and Semilinear Differential Inclusions in Banach Spaces, Wlter de Gruyter, Berlin, Germany, 2001.

[15] D. Bothe, "Multivalued perturbations of $m$-accretive differential inclusions," Israel Journal of Mathematics, vol. 108, 1998.

[16] B. C. Dhage, "Multi-valued mappings and fixed points II," Tamkang Journal of Mathematics, vol. 37, no. 1, pp. 27-46, 2006. 